\title{
ON COMMON FIXED POINTS OF COMMUTING CONTINUOUS FUNCTIONS ON AN INTERVAL
}

\author{
BY \\ JOHN PHILIP HUNEKE
}

This paper offers two methods of constructing commuting pairs of continuous functions (i.e. $f, g$ such that $f(g(x))=g(f(x))$ ) which map [0,1] to itself without common fixed points. Any such pair will be called "a solution to the commuting function problem".

\section{PART I}

LEMMA 1. Let $\left(f_{n} \mid n \in N\right),\left(g_{n} \mid n \in N\right)$ be two uniformly convergent sequences of continuous functions from $[0,1]$ to itself with limits $f, g$ respectively. If $f_{n} g_{n+1}$ $=g_{n} f_{n+1}$ for each $n \in N$, then $f, g$ commute.

LEMMA 2. Let h be a piecewise linear nowhere constant function (i.e. the derivative of $h$, denoted $D h$, is nowhere zero) defined on $I$; and let $A \subset h(I)$ be a finite set which contains the image under $h$ of all points in the interior of $I$ at which $D h$ does not exist. If $r, s$ are consecutive (with respect to the natural order on $R$ ) in $h^{-1}(A)$, then $h(r), h(s)$ are consecutive in $A$.

Proposition 1. Let $\left(f_{n} \mid n \in N\right),\left(g_{n} \mid n \in N\right)$ be sequences of piecewise linear functions from $[0,1]$ to itself such that either $\left|x-f_{2}(x)\right|>1 / 6$ or $\left|x-g_{2}(x)\right|>1 / 6$ for each $x \in[0,1]$. Then the limits of the sequences $\left(f_{n} \mid n \in N\right),\left(g_{n} \mid n \in N\right)$ form a solution to the commuting function problem provided that there exists a sequence $\left(A_{n} \mid n \in N\right)$ of finite subsets of $[0,1]$, and for each $n \in N$, the following properties are valid:

$P_{1}(n): f_{n} g_{n+1}=g_{n} f_{n+1}$;

$P_{2}(n):\left|D f_{n}(x)\right| \geqq 3$ and $\left|D g_{n}(y)\right| \geqq 3$ wherever the derivatives exist;

$P_{3}(n):$ if either $D f_{n}(x)$ or $D g_{n}(x)$ does not exist, then $x \in A_{n}$;

$P_{4}(n)$ : for each pair of consecutive points $r, s$ of $A_{n+1}$ with $r<s$,

$$
f_{n}([r, s])=f_{n+1}([r, s]) \text { and } g_{n}([r, s])=g_{n+1}([r, s])
$$

$P_{5}(n): f_{n}^{-1}\left(A_{n}\right)=A_{n+1}=g_{n}^{-1}\left(A_{n}\right) ;$ and $P_{6}(n): 0,1 \in A_{n}$.

Received by the editors July 28, 1967 and, in revised form, June 14, 1968. 
Proof. For any $m \in N^{+}$and for any pair of consecutive points $r, s$ of $A_{m+1}$, there exists by $P_{5}(n)$ and by Lemma 2 a pair of consecutive points $r^{\prime}, s^{\prime}$ of $A_{m}$ (equal to $g_{m}(r), g_{m}(s)$ respectively) such that $\frac{1}{3}\left|f_{m-1}\left(r^{\prime}\right)-f_{m-1}\left(s^{\prime}\right)\right| \geqq\left|f_{m}(r)-f_{m}(s)\right|$ since:

$$
\begin{aligned}
\frac{1}{3} \cdot\left|f_{m-1}\left(r^{\prime}\right)-f_{m-1}\left(s^{\prime}\right)\right| & =\frac{1}{3} \cdot\left|f_{m-1}\left(g_{m}(r)\right)-f_{m-1}\left(g_{m}(s)\right)\right| \\
& \left.=\frac{1}{3} \cdot\left|g_{m-1}\left(f_{m}(r)\right)-g_{m-1}\left(f_{m}(s)\right)\right| \quad \text { [by } P_{1}(m-1)\right] \\
& =\frac{1}{3} \cdot\left|f_{m}(r)-f_{m}(s)\right| \cdot \frac{\left|g_{m-1}\left(f_{m}(r)\right)-g_{m-1}\left(f_{m}(s)\right)\right|}{\left|f_{m}(r)-f_{m}(s)\right|} \\
& \geqq \frac{1}{3}\left|f_{m}(r)-f_{m}(s)\right| \cdot 3 \quad\left[\text { by } P_{5}(m), \text { Lemma 2, } P_{3}(m-1),\right. \text { and } \\
& =\left|f_{m}(r)-f_{m}(s)\right| .
\end{aligned}
$$

For every $n \in N$ and for every $x \in[0,1]$, there exist two consecutive points $r, s$ of $A_{n+1}$ such that $r \leqq x \leqq s$ (since $A_{n+1}$ is finite and contains 0,1 by $P_{6}(n+1)$ ), and so it follows that $\left|f_{n}(x)-f_{n+1}(x)\right| \leqq\left|f_{n}(r)-f_{n}(s)\right| \leqq(1 / 3)^{n}$ by $P_{4}(n), \quad P_{3}(n)$, and by iterated use of the result established in the preceding sentence. Therefore $\left\|f_{n}-f_{n+1}\right\|$ $\leqq 3^{-n}$. Since $\left(f_{n} \mid n \in N\right),\left(g_{n} \mid n \in N\right)$ have symmetric roles, we also have for each $n \in N\left\|g_{n}-g_{n+1}\right\| \leqq 3^{-n}$.

Now, $\left(f_{n} \mid n \in N\right),\left(g_{n} \mid n \in N\right)$ are uniformly convergent sequences, since for every pair $n, m \in N$ with $n \leqq m$, we have:

$$
\left\|f_{n}-f_{m}\right\| \leqq \sum_{i=n}^{+\infty}\left\|f_{i}-f_{i+1}\right\| \leqq \sum_{i=n}^{+\infty} 3^{-i}=\left(2 \cdot 3^{n-1}\right)^{-1}
$$

and similarly $\left\|g_{n}-g_{m}\right\| \leqq\left(2 \cdot 3^{n-1}\right)^{-1}$. From these inequalities, the limits of the two sequences of functions have no common fixed point since $\left\|f_{2}-\lim _{n \rightarrow \infty} f_{n}\right\| \leqq 1 / 6$ and $\left\|g_{2}-\lim _{n \rightarrow \infty} g_{n}\right\| \leqq 1 / 6$. Also, the limits commute by Lemma 1 .

Notation. If $f$ is a piecewise linear function defined on $I$, then let $B(f)$ denote the set of all points in the interior of $I$ at which $f$ has no derivative (i.e. at which $D f$ does not exist).

LEMMA 3. Let $f$ be a piecewise linear function defined on $I$; let $g$ be a function defined on $I$; let $h$ be a linear function with range $I$; and let 1 be a nonconstant linear function defined on $f(I)$. Then $g h(B(f h))=g(B(f)), B(1 f)=B(f)$, and $h^{-1}(B(f))=$ $B(f(h))$.

Definition. For any piecewise linear function $f$, if there exists an $s \in R^{+}$such that $|D f(x)|=s$ for each $x$ for which $D f(x)$ exists, then $f$ has a derivative of constant absolute value which we will denote by $f$ has DCAV; also, we will denote $s$ by slope $f$.

Notation. If $I$ is an interval with endpoints $r, s$, then let $T_{r s}: I \rightarrow I$ denote the linear function defined for each $x \in I$ by $T_{r s}(x)=r+s-x$.

Proposition 2. Let $f:\left[a, a^{\prime}\right] \rightarrow\left[b, b^{\prime}\right]$ and $g:\left[c, c^{\prime}\right] \rightarrow\left[b, b^{\prime}\right]$ be piecewise linear functions. If $\left\{f(a), f\left(a^{\prime}\right)\right\}=\left\{b, b^{\prime}\right\}=\left\{g(c), g\left(c^{\prime}\right)\right\}$ and if $f, g$ have DCAV, then there 
exist piecewise linear functions $j:\left[d, d^{\prime}\right] \rightarrow\left[a, a^{\prime}\right]$ and $k:\left[d, d^{\prime}\right] \rightarrow\left[c, c^{\prime}\right]$ satsifying the properties: $j(d)=a, j\left(d^{\prime}\right)=a^{\prime} ;\left\{k(d), k\left(d^{\prime}\right)\right\}=\left\{c, c^{\prime}\right\} ; j, k$ have $D C A V ; j(B(k))$ $\subset B(f), k(B(j)) \subset B(g)$; and $f j=g k$. The following diagram commutes:

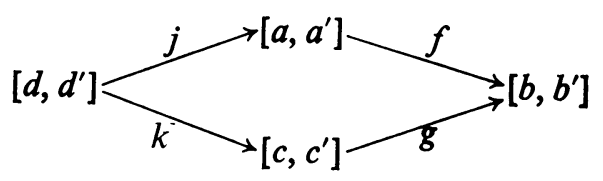

Proof. First Proposition 2 will be proved under the additional assumption that $f(a)=b=g(c)$ (and hence: $f\left(a^{\prime}\right)=b^{\prime}=g\left(c^{\prime}\right)$ ). We proceed by induction on the number of distinct points in $B(f) \cup B(g)$. Pick an interval $\left[d, d^{\prime}\right]$.

If $B(f) \cup B(g)$ is empty, then $f$ and $g$ are linear, so define $j, k$ to be the unique linear maps on $\left[d, d^{\prime}\right]$ such that $j(d)=a, j\left(d^{\prime}\right)=a^{\prime}, k(d)=c$, and $k\left(d^{\prime}\right)=c^{\prime}$. Observe that $f j$ and $g k$ are linear functions on $\left[d, d^{\prime}\right]$ such that $f j(d)=b=g k(d)$ and $f j\left(d^{\prime}\right)=$ $g k\left(d^{\prime}\right)$. Therefore $f j=g k$; also $B(j)$ and $B(k)$ are empty. So $j, k$ satisfy Proposition 2.

Now let $n \in N$ and assume the induction hypothesis: Proposition 2 is valid whenever $f(a)=b=g(c)$ and $B(f) \cup B(g)$ has no more than $n$ elements. Suppose we are given $f, g$ satisfying the hypothesis of Proposition 2 such that $f(a)=b=g(c)$ and $B(f) \cup B(g)$ has $n+1$ elements. Since $B(f) \cup B(g)$ is finite and nonempty, let $b^{\prime \prime}$ denote the smallest element of $f(B(f)) \cup g(B(g))$. Since $f$ and $g$ play symmetric roles, assume without loss of generality that $b^{\prime \prime} \in f(B(f))$. Let $a^{\prime \prime}$ denote any point in $B(f)$ such that $f\left(a^{\prime \prime}\right)=b^{\prime \prime} . B(f)$ equals the set of points which are local minimum or local maximum points for $f$ except for $a, a^{\prime}$, because $f$ has DVAC; also, $a^{\prime \prime}$ is a local minimum for $f$ since $f$ has a local maximum at the endpoint $a^{\prime}$ of $\left[a, a^{\prime}\right]$. Now $f$ has local minima at $a$ and $a^{\prime \prime}$, so $f$ must have a local maximum between $a$ and $a^{\prime \prime}$, i.e. $B\left(\left.f\right|_{\left[a, a^{\prime \prime}\right]}\right)$ is nonempty. Let $b^{\prime \prime}$ denote the maximum element of $f\left(B\left(\left.f\right|_{\left[a, a^{\prime \prime}\right]}\right)\right)$, and let $a^{\prime \prime \prime}$ be any element of $B\left(\left.f\right|_{\left[a, a^{\prime \prime}\right]}\right)$ such that $f\left(a^{m}\right)=b^{m}$. Let $c^{m}$ be the minimum element of $\left[c, c^{\prime}\right]$ such that $g\left(c^{\prime \prime \prime}\right)=b^{\prime \prime \prime}$, and let $c^{\prime \prime}$ be the maximum element of $\left[c, c^{\prime \prime \prime}\right]$ such that $g\left(c^{\prime \prime}\right)=b^{\prime \prime}$. Let $d^{\prime \prime}=\left(2 d+d^{\prime}\right) / 3$ and let $d^{\prime \prime \prime}=\left(d+2 d^{\prime}\right) / 3$. Observe that the induction hypothesis is applicable to each of the three pairs of functions: (i) $\left.f\right|_{\left[a, a^{\prime \prime \prime}\right]},\left.g\right|_{\left[c, c^{\prime \prime \prime}\right]}$; (ii) $f T_{a^{\prime \prime} a^{\prime \prime \prime}},\left.g\right|_{\left[c^{\prime \prime}, c^{\prime \prime \prime}\right]}$; and (iii) $\left.f\right|_{\left[a^{\prime \prime}, a^{\prime}\right]},\left.g\right|_{\left[c^{\prime \prime}, c^{\prime}\right]}$. Therefore, for (i) there exists $j^{\prime}, k^{\prime}$ defined on $\left[d, d^{\prime \prime}\right]$, for (ii) there exists $j^{\prime \prime}, k^{\prime \prime}$ defined on $\left[d^{\prime \prime}, d^{\prime \prime}\right]$, and for (iii) there exists $j^{\prime \prime \prime}, k^{m}$ defined on $\left[d^{m}, d^{\prime}\right]$ satisfying Proposition 2 (as stated for $f, g, j, k$ respectively). See Figure 1.

Now define the two points $d^{*}, d^{* *}$ :

$$
\begin{aligned}
d^{*} & =d+\left(d^{\prime}-d\right) \cdot \frac{\left(\text { slope } j^{\prime}\right)}{\left(\text { slope } j^{\prime}+\operatorname{slope} j^{\prime \prime}+\text { slope } j^{\prime \prime}\right)} \\
d^{* *} & =d+\left(d^{\prime}-d\right) \cdot \frac{\left(\text { slope } j^{\prime}+\text { slope } j^{\prime \prime}\right)}{\left(\text { slope } j^{\prime}+\operatorname{slope} j^{\prime \prime}+\operatorname{slope} j^{\prime \prime}\right)}
\end{aligned}
$$




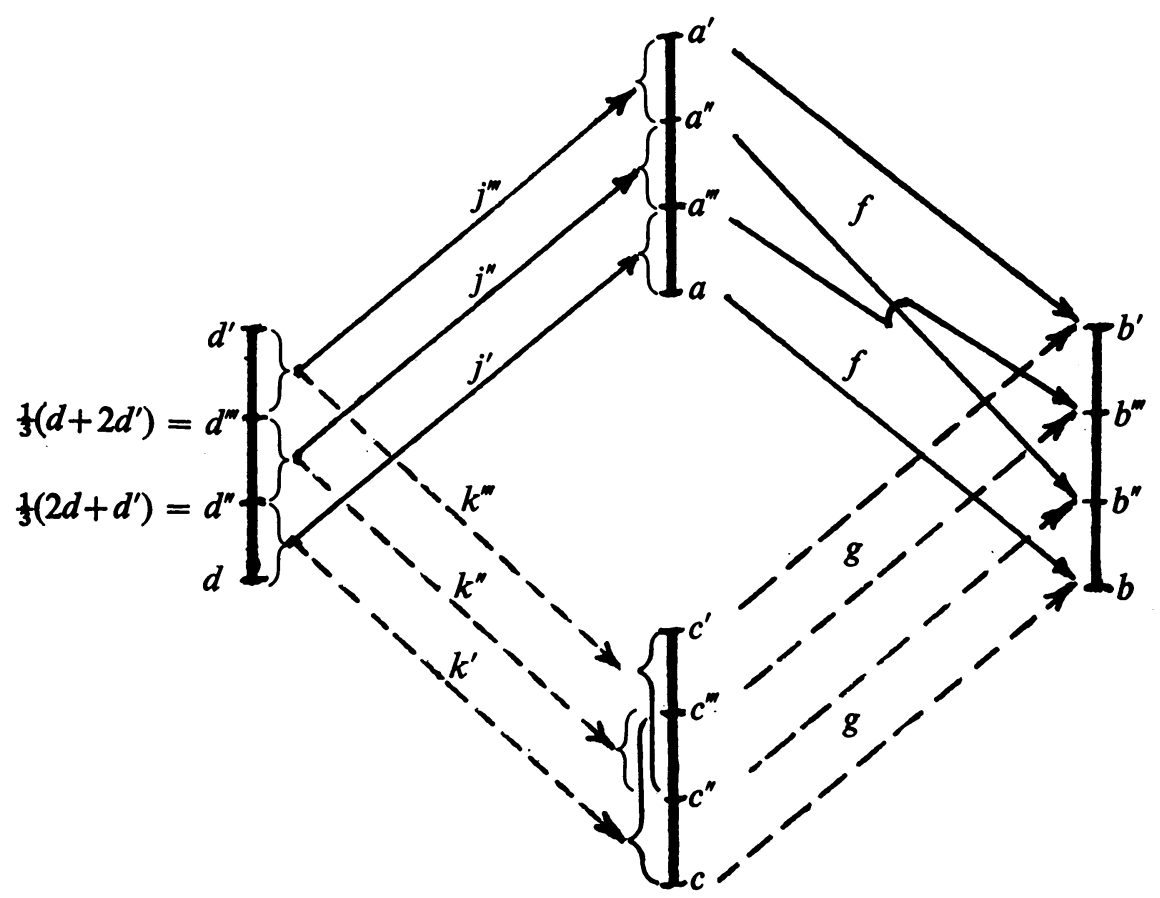

FIGURE 1. Induction step in the proof of Proposition 2.

Let $S^{\prime}$ be the linear function defined from $\left[d, d^{*}\right]$ onto $\left[d, d^{\prime \prime}\right]$ such that $S^{\prime}(d)=d$, let $S^{\prime \prime}$ be the linear function defined from $\left[d^{*}, d^{* *}\right]$ onto $\left[d^{\prime \prime}, d^{m}\right]$ such that $S^{\prime \prime}\left(d^{*}\right)$ $=d^{m}$, and let $S^{m}$ be the linear function defined from $\left[d^{* *}, d^{\prime}\right]$ onto $\left[d^{m}, d^{\prime}\right]$ such that $S^{\prime \prime}\left(d^{\prime}\right)=d^{\prime}$. Let $j=j^{\prime} S^{\prime} \cup T_{a^{\prime \prime} a^{\prime \prime \prime} j^{\prime \prime}} S^{\prime \prime} \cup j^{m} S^{m}$ (considering a function to be a set of ordered pairs), and let $k=k^{\prime} S^{\prime} \cup k^{\prime \prime} S^{\prime \prime} \cup k^{m} S^{m}$. By checking the points $d^{*}, d^{* *}$, it can be seen that $j, k$ are well defined functions (i.e. $j\left(d^{*}\right)=a^{m}, j\left(d^{* *}\right)=a^{\prime \prime}, k\left(d^{*}\right)=c^{m}$, and $\left.k\left(d^{* *}\right)=c^{\prime \prime}\right)$. It is clear that $j, k$ are piecewise linear functions. Also $j, k$ have DCAV with

slope $j=\frac{1}{3}\left(\right.$ slope $j^{\prime}+$ slope $j^{\prime \prime}+$ slope $\left.j^{\prime \prime}\right)$ and slope $k=\frac{1}{3}\left(\right.$ slope $k^{\prime}+$ slope $k^{\prime \prime}+$ slope $\left.k^{\prime \prime}\right)$.

Observe that $j(d)=a, j\left(d^{\prime}\right)=a^{\prime}, k(d)=c$, and $k\left(d^{\prime}\right)=c^{\prime}$. We also have:

$$
\begin{aligned}
j(B(k)) & \subset\left\{j\left(d^{*}\right), j\left(d^{* *}\right)\right\} \cup j\left(B\left(k^{\prime} S^{\prime}\right)\right) \cup j\left(B\left(k^{\prime \prime} S^{\prime \prime}\right)\right) \cup j\left(B\left(k^{m} S^{m \prime}\right)\right) \\
& =\left\{a^{\prime \prime}, a^{m}\right\} \cup j^{\prime} S^{\prime}\left(B\left(k^{\prime} S^{\prime}\right)\right) \cup T_{a^{\prime \prime} a^{\prime \prime \prime}} j^{\prime \prime} S^{\prime \prime}\left(B\left(k^{\prime \prime} S^{\prime \prime}\right)\right) \cup j^{m} S^{m}\left(B\left(k^{m} S^{m}\right)\right) \\
& =\left\{a^{\prime \prime}, a^{m}\right\} \cup j^{\prime}\left(B\left(k^{\prime}\right)\right) \cup T_{a^{\prime \prime} a^{\prime \prime \prime}} j^{\prime \prime}\left(B\left(k^{\prime \prime}\right)\right) \cup j^{m}\left(B\left(k^{m}\right)\right) \quad[\text { by Lemma 3] } \\
& \subset\left\{a^{\prime \prime}, a^{\prime \prime \prime}\right\} \cup B\left(\left.f\right|_{\left[a, a^{\prime \prime \prime}\right]}\right) \cup B\left(f T_{a^{\prime \prime} a^{\prime \prime \prime}} T_{\left.a^{\prime \prime} a^{\prime \prime \prime}-1\right) \cup B\left(\left.f\right|_{\left[a^{\prime \prime}, a^{\prime}\right]}\right)}\right. \\
& =B(f) .
\end{aligned}
$$

Therefore, $j(B(k)) \subset B(f)$. Now, since $j$ has DCAV, $x \in B(j)$ only if $x$ is either a 
local maximum or a local minimum of $j$. Neither $d^{*}$ nor $d^{* *}$ is a local maximum or a local minimum of $j$, so neither is in $B(j)$. So:

$$
\begin{aligned}
& k(B(j))=k\left(B\left(j^{\prime} S^{\prime}\right)\right) \cup k\left(B\left(T_{a^{\prime \prime} a^{\prime \prime} j^{\prime \prime}} S^{\prime \prime}\right)\right) \cup k\left(B\left(j^{m} S^{m}\right)\right) \\
& =k^{\prime} S^{\prime}\left(B\left(j^{\prime} S^{\prime}\right)\right) \cup k^{\prime \prime} S^{\prime \prime}\left(B\left(T_{a^{\prime \prime} a^{\prime \prime \prime}} j^{\prime \prime} S^{\prime \prime}\right)\right) \cup k^{m} S^{m}\left(B\left(j^{m} S^{m}\right)\right) \\
& =k^{\prime}\left(B\left(j^{\prime}\right)\right) \cup k^{\prime \prime}\left(B\left(j^{\prime \prime}\right)\right) \cup k^{m}\left(B\left(j^{\prime \prime}\right)\right) \quad \text { [by Lemma 3] } \\
& \subset B\left(\left.g\right|_{\left[c, c^{\prime \prime \prime}\right]}\right) \cup B\left(\left.g\right|_{\left[c^{\prime \prime}, c^{\prime \prime \prime}\right]}\right) \cup B\left(\left.g\right|_{\left[c^{\prime \prime}, c^{\prime}\right]}\right) \\
& \subset B(g) \text {. }
\end{aligned}
$$

Therefore, $k(B(j)) \subset B(g)$. It is clear that $f j=g k$. Hence, $j$; $k$ satisfy Proposition 2 . By induction, Proposition 2 has been proved provided $f(a)=b=g(c)$.

Now assume that $f, g$ satisfy the hypothesis of Proposition 2.

CASE 1. If $f(a)=b=g(c)$, then $j, k$ exist satisfying Proposition 2 as defined above.

CASE 2. If $f(a)=b=g\left(c^{\prime}\right)$, then apply Case 1 to $f, g T_{c c^{\prime}}$ to get two functions $j, k$; now $j, T_{c c^{\prime}} k$ satisfy Proposition 2 .

CASE 3. If $f(a)=b^{\prime}=g\left(c^{\prime}\right)$, then apply Case 1 to $f T_{a a^{\prime}}, g$ to get two functions $j, k$; now $T_{a a^{\prime}} j T_{d d^{\prime}}, k T_{d d^{\prime}}$ satisfy Proposition 2 .

CASE 4. If $f(a)=b^{\prime}=g(c)$, then apply Case 1 to $f T_{a a^{\prime}}, g T_{c c^{\prime}}$ to get two functions $j, k$; now $T_{a a^{\prime}} j T_{d d^{\prime}}, T_{c c^{\prime}} k T_{d d^{\prime}}$ satisfy Proposition 2 .

This completes the proof of Proposition 2.

LEMMA 4. Let the two functions $h, k$ map $\left[a, a^{\prime}\right]$ onto $\left[b, b^{\prime}\right]$ and let $h$ be linear and $k$ be piecewise linear. If $k$ has $D C A V$, then slope $h \leqq$ slope $k$.

The Construction. We are now ready to construct (using Proposition 2) sequences $\left(f_{n} \mid n \in N\right),\left(g_{n} \mid n \in N\right),\left(A_{n} \mid n \in N\right)$ which satisfy the conditions of Proposition 1. Specifically we desire two sequences $\left(f_{n} \mid n \in N\right),\left(g_{n} \mid n \in N\right)$ of piecewise linear functions mapping $[0,1]$ into itself such that for every $x \in[0,1]$ either $\left|x-f_{2}(x)\right|>\frac{1}{6}$ or $\left|x-g_{2}(x)\right|>\frac{1}{6}$, and a sequence $\left(A_{n} \mid n \in N\right)$ of finite subsets of $[0,1]$ such that $0,1 \in A_{0}$, and such that the following properties are satisfied for every $n \in N$ :

$P_{i}(n)$ : for $i=1,2,3,4,5$ as in Proposition 1;

$P_{7}(n):\left.f_{n}\right|_{A_{n+1}}=\left.f_{n+1}\right|_{A_{n+1}}$ and $\left.g_{n}\right|_{A_{n+1}}=\left.g_{n+1}\right|_{A_{n+1}}$;

$P_{8}(n): f_{n}\left(B\left(g_{n}\right)\right) \cup g_{n}\left(B\left(f_{n}\right)\right) \subset A_{n}$;

$P_{9}(n): A_{n} \subset A_{n+1}$; and,

$P_{10}(n)$ : for all $r^{\prime}, s^{\prime}$ consecutive in $A_{n}$ such that $r^{\prime}<s^{\prime}$, $\left.f_{n}\right|_{\left[r^{\prime}, s^{\prime}\right]}$ and $\left.g_{n}\right|_{\left[r^{\prime}, s^{\prime}\right]}$ each have DCAV.

Now define $A_{0}=\{0,1\}, A_{1}=\{0,1 / 3,2 / 3,1\}$, and

$$
A_{2}=\{0,1 / 9,2 / 9,1 / 3,6 / 15,7 / 15,8 / 15,9 / 15,2 / 3,7 / 9,8 / 9,1\} .
$$

Also, define $f_{0}, g_{0}, f_{1}, g_{1}, f_{2}$, and $g_{2}$ at each $x \in[0,1]$ as follows:

if $0 \leqq x<1 / 3$, let $f_{0}(x)=3 x$, and let $g_{0}(x)=1-3 x$;

if $1 / 3 \leqq x<2 / 3$, let $f_{0}(x)=2-3 x$, and let $g_{0}(x)=3 x-1$; 
if $2 / 3 \leqq x \leqq 1$, let $f_{0}(x)=3 x-2$, and let $g_{0}(x)=3-3 x$;

if $0 \leqq x<1 / 3$ or $2 / 3 \leqq x \leqq 1$, let $f_{1}(x)=f_{0}(x)$, and let $g_{1}(x)=g_{0}(x)$;

if $1 / 3 \leqq x<6 / 15$, let $f_{1}(x)=(8 / 3)-5 x$;

if $6 / 15 \leqq x<7 / 15$, let $f_{1}(x)=5 x-(4 / 3)$;

if $7 / 15 \leqq x<2 / 3$, let $f_{1}(x)=(10 / 3)-5 x$;

if $1 / 3 \leqq x<8 / 15$, let $g_{1}(x)=5 x-(5 / 3)$;

if $8 / 15 \leqq x<9 / 15$, let $g_{1}(x)=(11 / 3)-5 x$;

if $9 / 15 \leqq x<2 / 3$, let $g_{1}(x)=5 x-(7 / 3)$;

if $0 \leqq x<6 / 15$ or $7 / 15 \leqq x \leqq 1$, let $f_{2}(x)=f_{1}(x)$;

if $6 / 15 \leqq x<31 / 75$, let $f_{2}(x)=(25 / 3) x-(8 / 3)$;

if $31 / 75 \leqq x<32 / 75$, let $f_{2}(x)=(38 / 9)-(25 / 3) x$;

if $32 / 75 \leqq x<7 / 15$, let $f_{2}(x)=(25 / 3) x-(26 / 9)$;

if $0 \leqq x<8 / 15$ or $9 / 15 \leqq x \leqq 1$, let $g_{2}(x)=g_{1}(x)$;

if $8 / 15 \leqq x<41 / 75$, let $g_{2}(x)=(49 / 9)-(25 / 3) x$;

if $41 / 75 \leqq x<42 / 75$, let $g_{2}(x)=(25 / 3) x-(11 / 3)$; and

if $42 / 75 \leqq x<9 / 15$, let $g_{2}(x)=(17 / 3)-(25 / 3) x$.

Observe that $f_{i}, g_{i}, A_{i}$ for $i=0,1,2$ have been defined satisfying the desired properties. By Figure 2 for each $x \in[0,1],\left|x-f_{2}(x)\right|>1 / 6$ or $\left|x-g_{2}(x)\right|>1 / 6$. Also, the properties $P_{1}(i-1), P_{2}(i), P_{3}(i-1), P_{4}(i-1), P_{5}(i-1), P_{7}(i-1), P_{8}(i), P_{9}(i-1)$, and $P_{10}(i)$ are satisfied for $i=1,2$.

Proceed to define $\left(f_{n} \mid n \in N\right),\left(g_{n} \mid n \in N\right)$, and $\left(A_{n} \mid n \in N\right)$ by induction; let $n \in N, n \geqq 2$, and assume that $f_{i}, g_{i}$, and $A_{i}$ (for $i=0,1,2, \ldots, n$ ) have been defined satisfying the desired properties, especially: $P_{1}(i-1), P_{2}(i), P_{3}(i-1), P_{4}(i-1)$, $P_{5}(i-1), P_{7}(i-1), P_{8}(i), P_{9}(i-1)$, and $P_{10}(i)$ for $i=1,2, \ldots, n$. Define $A_{n+1}$ $=f_{n}^{-1}\left(A_{n}\right)$. Notice that

$$
A_{n+1}=f_{n}^{-1}\left(A_{n}\right)=f_{n}^{-1} g_{n-1}^{-1}\left(A_{n-1}\right)=g_{n}^{-1} f_{n-1}^{-1}\left(A_{n-1}\right)=g_{n}^{-1}\left(A_{n}\right),
$$

so $P_{5}(n)$ is satisfied. For each $x \in[0,1]$ define $f_{n+1}(x)$ and $g_{n+1}(x)$ in the following manner. There exist two consecutive points $d, d^{\prime}$ of $A_{n+1}$ such that $d \leqq x \leqq d^{\prime}$ (since $A_{n+1}$ is finite and contains 0,1$)$. We can set $\left\{a, a^{\prime}\right\}=\left\{g_{n}(d), g_{n}\left(d^{\prime}\right)\right\},\left\{b, b^{\prime}\right\}$ $=\left\{f_{n-1} g_{n}(d), f_{n-1} g_{n}\left(d^{\prime}\right)\right\}$, and $\left\{c, c^{\prime}\right\}=\left\{f_{n}(d), f_{n}\left(d^{\prime}\right)\right\}$ such that $a<a^{\prime}, b<b^{\prime}$, and $c<c^{\prime}$ by Lemma 2. Now observe that $\left.f_{n}\right|_{\left[a, a^{\prime}\right]},\left.g_{n}\right|_{\left[c, c^{\prime}\right]}$ satisfy the hypothesis of Proposition 2 for $f, g$ respectively; so let $j, k$ be defined by Proposition $2, j$ mapping $\left[d, d^{\prime}\right]$ onto $\left[a, a^{\prime}\right]$ and $k$ mapping $\left[d, d^{\prime}\right]$ onto $\left[c, c^{\prime}\right]$. If $g_{n}(d)=a$, then define $f_{n+1}(x)=k(x)$, and $g_{n+1}(x)=j(x)$; if $g_{n}(d)=a^{\prime}$, then define $f_{n+1}(x)=k T_{d d^{\prime}}(x)$, and $g_{n+1}(x)=j T_{d d^{\prime}}(x)$. In the above procedure, for a fixed $x \in[0,1]$, the points $d, d^{\prime}$ (defined to be consecutive points in $A_{n+1}$ such that $d \leqq x \leqq d^{\prime}$ ) are not necessarily unique. However, $f_{n+1}$ and $g_{n+1}$ are well defined functions since $\left.g_{n+1}\right|_{A_{n+1}}=\left.g_{n}\right|_{A_{n+1}}$ by definition, and hence $\left.f_{n+1}\right|_{A_{n+1}}=\left.f_{n}\right|_{A_{n+1}}$. Therefore $P_{7}(n)$ is satisfied. $P_{1}(n)$ is satisfied since for each $x$ in $[0,1]$ there exist $d, d^{\prime}$ consecutive in $A_{n+1}$ such that $d \leqq x \leqq d^{\prime}$ and either $f_{n} g_{n+1}(x)=f_{n} j T_{d d^{\prime}}(x)=g_{n} k T_{d d^{\prime}}(x)=g_{n} f_{n+1}(x)$, or $f_{n} g_{n+1}(x)=f_{n} j(x)=$ $g_{n} k(x)=g_{n} f_{n+1}(x)$ where $j, k$ are defined by Proposition 2 as above; hence $f_{n} g_{n+1}$ 


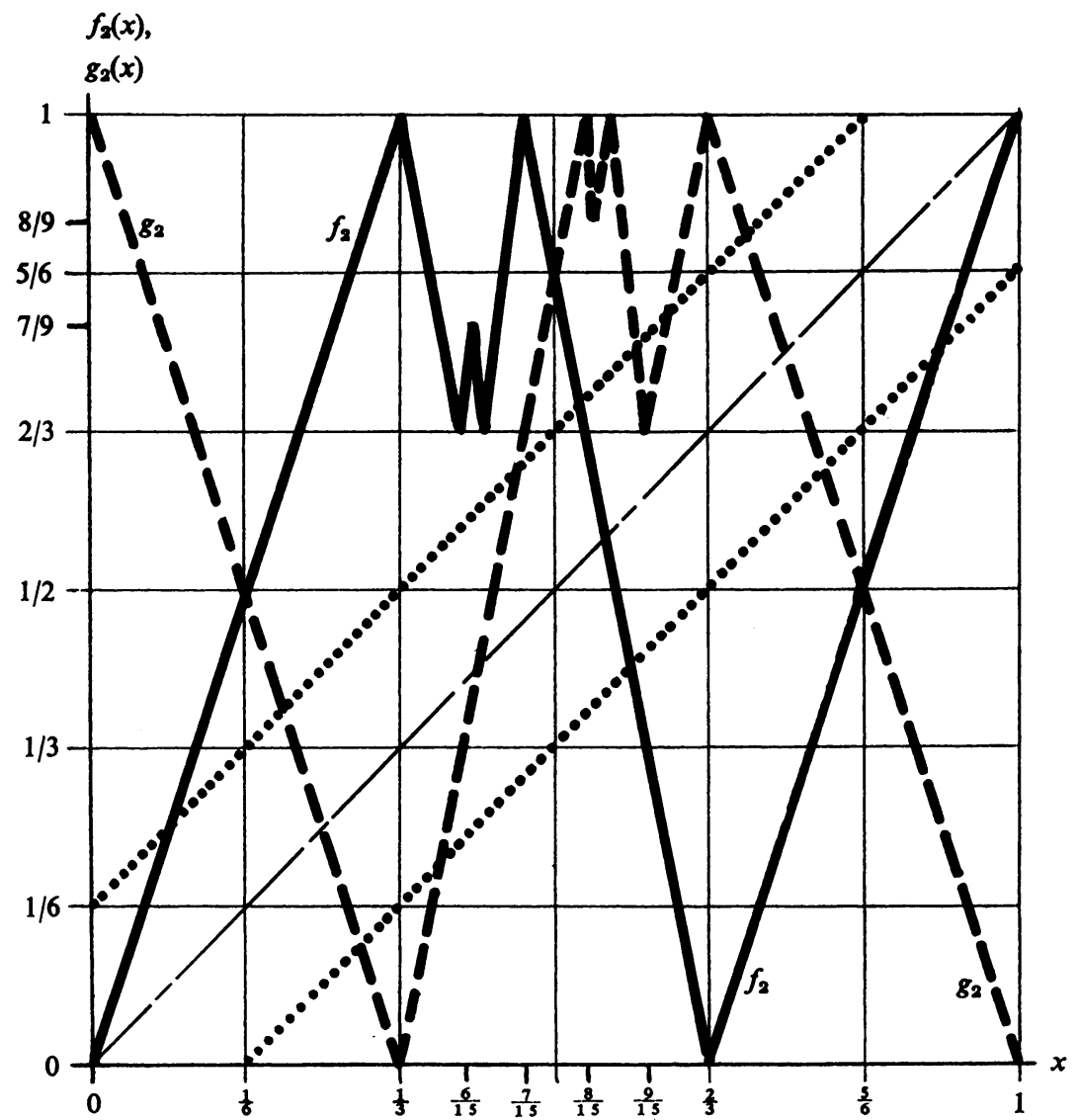

Figure 2. The graphs of $f_{2}, g_{2}$ and the diagonal; $f_{2}$ or $g_{2}$ lies between the dotted lines wherever $\left|x-f_{2}(x)\right| \leqq \frac{1}{6}$ or $\left|x-g_{2}(x)\right| \leqq \frac{1}{6}$ respectively.

$=g_{n} f_{n+1} . P_{3}(n)$ is satisfied by $P_{5}(n)$ and $P_{8}(n) . P_{4}(n)$ is satisfied by $P_{3}(n)$ and Proposition 2. $P_{10}(n+1)$ is satisfied by Proposition 2. $P_{2}(n+1)$ is satisfied by $P_{2}(n), P_{3}(n)$, $P_{4}(n), P_{10}(n+1)$, and Lemma $4 . P_{9}(n)$ is satisfied by $P_{5}(n-1), P_{7}(n-1), P_{9}(n-1)$, and $P_{5}(n) . P_{8}(n+1)$ is satisfied by Proposition 2, Lemma $3, P_{5}(n), P_{9}(n)$, and $P_{3}(n)$. Therefore $f_{i}, g_{i}$, and $A_{i}$ (for $i=0,1,2, \ldots, n, n+1$ ) have been defined satisfying the desired properties, especially: $P_{1}(i-1), P_{2}(i), P_{3}(i-1), P_{4}(i-1), P_{5}(i-1), P_{7}(i-1)$, $P_{8}(i), P_{9}(i-1)$, and $P_{10}(i)$ for $i=1,2, \ldots, n, n+1$.

Therefore, the sequences $\left(f_{n} \mid n \in N\right),\left(g_{n} \mid n \in N\right)$, and $\left(A_{n} \mid n \in N\right)$ have been defined by induction satisfying Proposition 1. So $\lim _{n \rightarrow \infty} f_{n}$ and $\lim _{n \rightarrow \infty} g_{n}$ form a solution to the commuting function problem.

REMARK. Simultaneous to and independent of the author's preceding work $\left({ }^{1}\right)$, W. M. Boyce [1], [2] constructed essentially the same solution to the commuting

(1) Compare [1] and [3]. 
function problem defined above. These functions are nowhere Lipschitzian; smoother solutions are described in Part II below.

\section{PART II}

Notation. For any real valued mapping $h$ defined on a subset of the reals, let $h^{*}$ denote the map: $h^{*}(x)=1-h(1-x)$ for each $x$ for which $h(1-x)$ is defined; also, $h$ will be called $s$-Lipschitzian provided $s$ is a real number and for each $x, y$ in the domain of $h,|h(x)-h(y)| \leqq s \cdot|x-y|$. Now pick any $b$ in $\left[0, \frac{1}{2}\right]$, and define

$$
s=\frac{3-2 b+(6-4 b)^{1 / 2}}{1-2 b}
$$

Define the three linear functions:

$h_{1}:[b,(1-b+s b) / s] \rightarrow[b, 1]$ by $h_{1}(x)=s x-s b+b$;

$h_{2}:[(1-b+s b) / s,(2-b+s b) / s] \rightarrow[0,1]$ by $h_{2}(x)=2-s x+s b-b$;

$h_{3}:[(2-b+s b) / s,(3-2 b+s b) / s] \rightarrow[0,1-b]$ by $h_{3}(x)=-2+s x-s b+b$.

And define the piecewise linear function $h:[b,(3-2 b+s b) / s] \rightarrow[0,1]$ by $h=h_{1}$ $\cup h_{2} \cup h_{3}$. Let $C_{b}$ denote all continuous functions from $[0, b]$ to $[0, b]$ which have $b$ as a fixed point.

Definition. For each $g$ in $C_{b}$ let $\bar{g}$ denote the unique extension of $g$ defined by:

1. $\bar{g}(x)=g(x)$ whenever $0 \leqq x \leqq b$;

2. $\bar{g}(x)=h(x)$ whenever $b \leqq x \leqq h_{3}^{-1}(1-b)$;

3. $\bar{g}(x)=h_{1}^{*-1} \bar{g}\left(h^{*}(x)\right)$ whenever $h_{3}^{-1}(1-b) \leqq x \leqq h_{2}^{*-1}\left(h_{2}^{-1}(0)\right)$;

4. $\bar{g}(x)=h_{2}^{*-1} \bar{g}\left(h^{*}(x)\right)$ whenever $h_{2}^{*-1}\left(h_{2}^{-1}(0)\right) \leqq x \leqq 1-b$; and

5. $\bar{g}(x)=$ the fixed point of $h_{2}^{*}$ whenever $1-b \leqq x \leqq 1$.

See Figure 3.

REMARK. That the preceding definition is consistent can be checked by direct mechanical methods, or (as suggested by Felix Albrecht) by a Zorn's Lemma argument. The following sketch of a proof that $\bar{g}$ is uniquely defined above for any $s$-Lipschitzian $g$ in $C_{b}$ was suggested by David Boyd.

Proof. Let $g$ be an $s$-Lipschitzian function in $C_{b}$, and let $L$ denote the set of $s$-Lipschitzian functions from $[0,1]$ to itself which satisfy properties 1,2 , and 5 for $\bar{g}$ in the Definition. Now define the mapping $T$ from $L$ to $L$ : for any $f$ in $L$, let

$T(f)(x)=f(x)$ whenever $0 \leqq x \leqq h_{3}^{-1}(1-b)$ or whenever $1-b \leqq x \leqq 1$;

$T(f)(x)=h_{1}^{*-1} f\left(h^{*}(x)\right)$ whenever $h_{3}^{-1}(1-b) \leqq x \leqq h_{2}^{*-1}\left(h_{2}^{-1}(0)\right)$;

$T(f)(x)=h_{2}^{*-1} f\left(h^{*}(x)\right)$ whenever $h_{2}^{*-1}\left(h_{2}^{-1}(0)\right) \leqq x \leqq 1-b$.

To see that $T(f)$ is in $L$, observe that $T(f)$ is $s$-Lipschitzian by a system of inequalities using the facts that $h_{1}^{*-1}$ and $h_{2}^{*-1}$ are linear and $1 / s$-Lipschitzian, $h_{1}^{*-1}(0)$ $=h_{2}^{*-1}(0), h^{*}$ is $s$-Lipschitzian, and the fixed points of $h^{*}$ are $h_{3}^{-1}(1-b)$, $(s-1)(1-b) /(s+1)$, and $1-b$.

$L$ is clearly a complete metric space with respect to the supremum norm metric, and $T$ is a contraction of this metric space with constant $1 / s$ (since $h_{1}^{*-1}$ and $h_{2}^{*-1}$ 


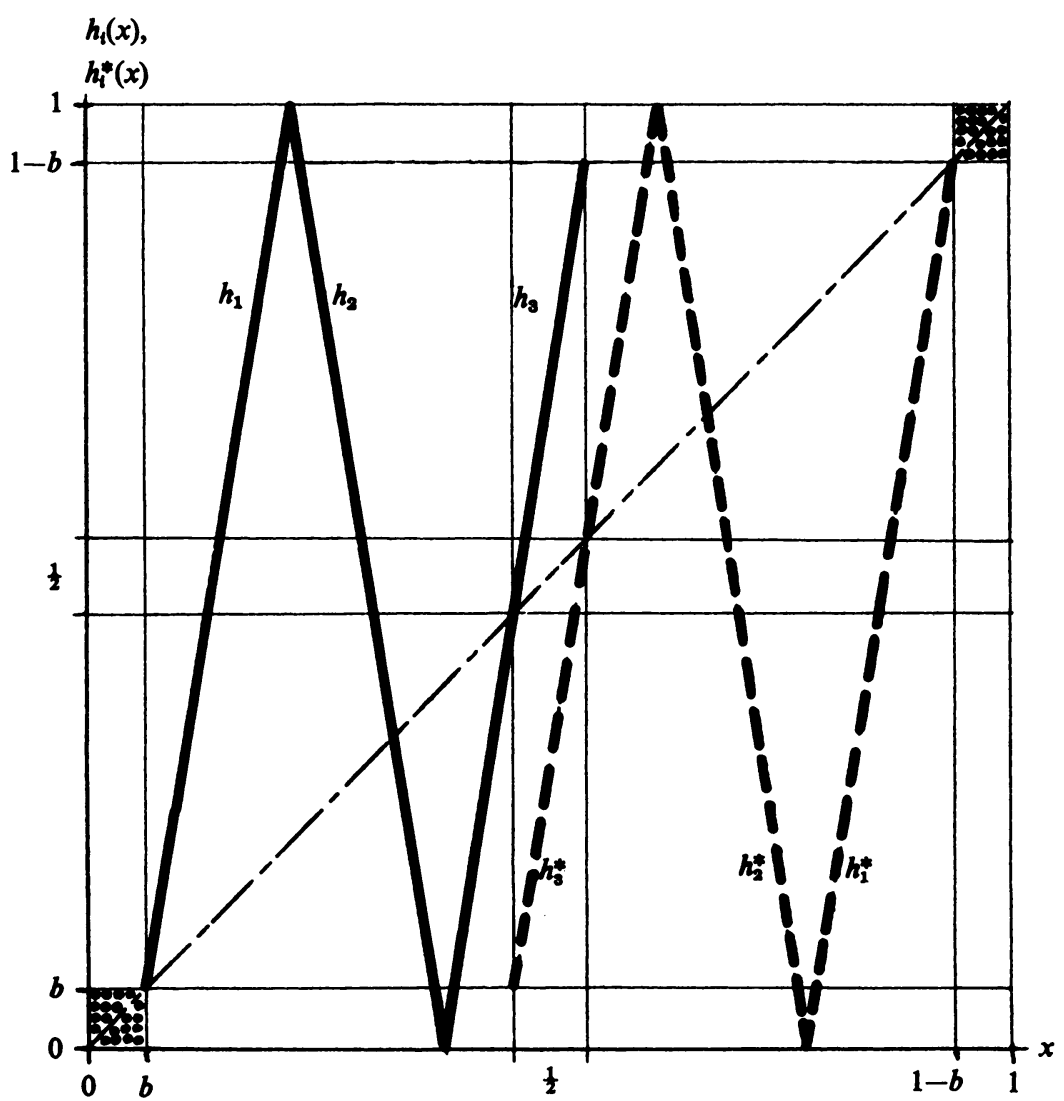

FIGURE 3. Graphs of $h_{i}, h_{i}^{*}(i=1,2,3)$ with $b=3 / 50$ and $s=6$. The solid line is the graph of $h$; the dashed line is the graph of $h^{*}$. The dotted regions contain the graphs of $g$ and $g^{*}$ for any $g$ in $C_{b}$.

are $1 / s$-Lipschitzian). Hence there is a unique function $\bar{g}$ in $L$ such that $T(\bar{g})=\bar{g}$, and this function satisfies the definition of $\bar{g}$.

Lemma 5. For any $f, g$ in $C_{b}$ and any $x$ in $\left[\frac{1}{2}, 1\right]$,

(1) $\bar{f}^{*}(x)=x$ implies $\bar{g}(x) \neq x$, and

(2) $\bar{f}^{*}(\bar{g}(x))=\bar{g}\left(\bar{f}^{*}(x)\right)$.

Proof of (1). As a comment to clarify notation, $\bar{f}^{*}(x)=(\bar{f})^{*}(x)$. Observe that the domain of definition of $h^{*}$ is $\left[h_{3}^{*-1}(b), 1-b\right]$, and

$$
h_{3}^{*-1}(b)=\frac{-3+2 b+s-s b}{s}=1-b+\frac{(1-2 b)(2 b-3)}{3-2 b+6-4 b}<\frac{1}{2},
$$

since $0 \leqq b<\frac{1}{2}$. So on $\left[\frac{1}{2}, 1\right]$, the fixed points of $\bar{f}^{*}$ are either in $[1-b, 1]$ or are the fixed points of $h^{*}$. By definition of $\bar{g}$ on $[1-b, 1], \bar{g}(x)$ equals the fixed point of $h_{2}^{*}$ which equals $(1-b)(s-1) /(1+s)<1-b \leqq x$ for each $x \in[1-b, 1]$. Therefore $\bar{g}$ 
and $\bar{f}^{*}$ have no common fixed point in $[1-b, 1]$. The only fixed points of $h^{*}$ are the fixed points of $h_{i}^{*}$ for $i=1,2,3$. The fixed point of $h_{1}^{*}$ is $1-b$, which (as has just been seen) is not a fixed point of $\bar{g}$. Denote the fixed point of $h_{2}^{*}$ by $x_{2}$;

$$
x_{2}=\frac{(1-b)(s-1)}{s+1}<\frac{b-2+s+s^{2}-s^{2} b-2 s}{s^{2}}=h_{2}^{*-1}\left(h_{2}^{-1}(0)\right) \text {, }
$$

and so

$$
\bar{g}\left(x_{2}\right) \in h_{1}^{*-1}([0,1])=[(s-1+b-s b) / s,(s+b-s b) / s] .
$$

However,

$$
x_{2}=\frac{(1-b)(s-1)}{1+s}<\frac{s-1+b-s b}{s} \leqq \bar{g}\left(x_{2}\right),
$$

so $x_{2}$, the fixed point of $h_{2}^{*}$, is not a fixed point for $\bar{g}$. Let $x_{3}$ denote the fixed point of $h_{3}^{*}$;

$$
x_{3}=\frac{3-s+s b-b}{1-s}=\frac{3-2 b+s b}{s}=h_{3}^{-1}(1-b) .
$$

Therefore $\bar{g}\left(x_{3}\right)=g\left(h_{3}^{-1}(1-b)\right)=1-b>x_{3}$. Therefore $\bar{f}^{*}$ and $\bar{g}$ have no common fixed point in $\left[\frac{1}{2}, 1\right]$.

Proof of (2). For each $x \in[1-b, 1], \bar{f}^{*}(\bar{g}(x))=\bar{f}^{*}\left(x_{2}\right)=h_{2}^{*}\left(x_{2}\right)=x_{2}$, and $\bar{g}\left(\bar{f}^{*}(x)\right)$ $=\bar{g}(1-\bar{f}(1-x))=\bar{g}(1-f(1-x))=x_{2}$ since $f(1-x) \in[0, b]$. Therefore $\bar{f}^{*}(\bar{g}(x))$ $=\bar{g}\left(\bar{f}^{*}(x)\right)$ for each $x \in[1-b, 1]$. For each $x \in\left[h_{2}^{*-1}\left(h_{2}^{-1}(0)\right), 1-b\right]$,

$$
\bar{f}^{*}(\bar{g}(x))=\bar{f}^{*}\left(h_{2}^{*-1} \bar{g}\left(h^{*}(x)\right)\right)=h_{2}^{*} h_{2}^{*-1} \bar{g} h^{*}(x)=\bar{g} \bar{f}^{*}(x) ;
$$

hence, $\bar{f}^{*}(\bar{g}(x))=\bar{g}\left(\bar{f}^{*}(x)\right)$. For each $x \in\left[h_{3}^{-1}(1-b), h_{2}^{*-1}\left(h_{2}^{-1}(0)\right)\right]$,

$$
\bar{f}^{*}(\bar{g}(x))=\bar{f}^{*}\left(h_{1}^{*-1} \bar{g}\left(h^{*}(x)\right)\right)=h_{1}^{*} h_{1}^{*-1} \bar{g}\left(h^{*}(x)\right)=\bar{g}\left(\bar{f}^{*}(x)\right) ;
$$

hence $\bar{f}^{*}(\bar{g}(x))=\bar{g}\left(\bar{f}^{*}(x)\right)$. Now $h_{3}^{-1}(1-b)$ is the fixed point of $h_{3}^{*}$ (as has been seen above), so

$$
h_{3}^{*}\left(\left[h_{3}^{*-1}(b), h_{3}^{-1}(1-b)\right]\right)=\left[b, h_{3}^{-1}(1-b)\right]
$$

which equals the domain of definition of $h$; also $h_{3}^{*-1}(1-b)$ is the fixed point of $h_{3}$, so

$$
h_{3}\left(\left[h_{3}^{*-1}(b), h_{3}^{-1}(1-b)\right]\right)=\left[h_{3}^{*-1}(b), 1-b\right]
$$

which equals the domain of definition of $h^{*}$. Observe that the two piecewise linear functions

$$
h^{*}\left(\left.h\right|_{\left[h_{3}^{*-1}(b), h_{3}^{-1}(1-b)\right]}\right), \quad\left(h^{*}\left(\left.h\right|_{\left[h_{3}^{*-1}(b), h_{3}^{-1}(1-b)\right]}\right)\right)^{*}
$$

are each the union of three linear functions and map the points $h_{3}^{*-1}(b)$, $h_{3}^{-1}\left(h^{*-1}(1)\right), h_{3}^{-1}\left(h^{*-1}(0)\right), h_{3}^{-1}(1-b)$ to $b, 1,0,1-b$ respectively; hence the two functions coincide. Therefore for each $x \in\left[\frac{1}{2}, h_{3}^{-1}(1-b)\right], x \in\left[h_{3}^{*-1}(b), h_{3}^{-1}(1-b)\right]$, so

$$
\begin{aligned}
\bar{f}^{*}(\bar{g}(x)) & =\bar{f}^{*}\left(h_{3}(x)\right)=h^{*} h(x)=\left(h^{*} h\right)^{*}(x)=1-h^{*}(h(1-x)) \\
& =h(1-h(1-x))=h\left(h^{*}(x)\right)=g\left(h_{3}^{*}(x)\right)=\bar{g}\left(\bar{f}^{*}(x)\right) .
\end{aligned}
$$

Therefore $\bar{f}^{*}$ and $\bar{g}$ commute on $\left[\frac{1}{2}, 1\right]$ and have no common fixed point in $\left[\frac{1}{2}, 1\right]$. 
Proposition 3. For any $f, g$ in $C_{b}, \bar{f}$ and $\bar{g}^{*}$ form a solution to the commuting function problem.

Proof. Let $f, g$ be in $C_{b}$. Then by Lemma $5, \bar{f}$ and $\bar{g}^{*}$ commute without common fixed point on $\left[\frac{1}{2}, 1\right]$; also $\bar{f}^{*}$ and $\bar{g}$ commute without common fixed point on $\left[\frac{1}{2}, 1\right]$. Therefore $\bar{f}^{* *}$ and $\bar{g}^{*}$ commute without common fixed point on $\left[0, \frac{1}{2}\right]$. But $\bar{f}^{* *}=\bar{f}$, so $\bar{f}$ and $\bar{g}^{*}$ form a solution to the commuting function problem.

Corollary. If $f, g$ are in $C_{b}$, then:

(1) $\bar{f}, \bar{g}^{*}$ form a solution to the commuting function problem;

(2) $\bar{f}, \bar{g}^{*}$ are s-Lipschitzian if and only if $f, g$ are s-Lipschitzian;

(3) $\bar{f}, \bar{g}^{*}$ are linear on each component of a dense open subset of $[0,1]$ if and only if $f, g$ are linear on each component of a dense open subset of $[0, b]$; and

(4) $\bar{f}, \bar{g}^{*}$ are differentiable almost everywhere if and only if $f, g$ are differentiable almost everywhere.

\section{REFERENCES}

1. W. M. Boyce, Commuting functions with no common fixed point, Abstract 67T-218, Notices Amer. Math. Soc. 14 (1967), 280.

2. - Commuting functions with no common fixed point, Trans. Amer. Math. Soc. 137 (1969), 77-92.

3. J. P. Huneke, Two counterexamples to a conjecture on commuting continuous functions of the closed unit interval, Abstract 67T-231, Notices Amer. Math. Soc. 14 (1967), 284.

WESLEYAN UNIVERSITY, Middletówn, ConNecticut

UNIVERSITY OF MINNESOTA, MinNeAPolis, MinNesota 